\title{
Nonlocal multi-point in time Cauchy problem for parabolic equations with degeneration
}

\author{
I. D. Pukal'skii, B. O. Yashan
}

Yuriy Fedkovych Chernivtsi National University, Chernivtsi, Ukraine
Corresponding author. E-mail: bohdanjaschan94@gmail.com

Paper received 07.05.20; Accepted for publication 29.05.20.

\section{https://doi.org/10.31174/SEND-NT2020-233VIII28-09}

Abstract. A multi-point in time problem for a parabolic equation with power singularities of arbitrary order in coefficients for time and spatial variables is considered. The existence and uniqueness of the solutions of the problem in the Hölder spaces with degree weight are established.

Keywords: degeneration, impulse action, apriori estimation.

Introduction. Nonlocal multi-point in time problem applies to nonlocal boundary value problems for partial differential equations whose theory is developed intensively since the seventies of the last century. The study of such problems is conditioned by many applications in the fields of mechanics, physics, chemistry, biology, ecology and other natural sciences, that arise in mathematical modeling of various processes [1, 2, 3].

At present, the theory of equations with degenerations and singularities is extensive field of mathematical knowledge with many areas of scientific research. It takes an important place in the theory of partial differential equations, as at the expense of many analytical results, and thanks to numerous applications in various sections of mathematics and physics $[4,5]$.

This article suggests an algorithm for solving the nonlocal multi-point in time problem for the parabolic secondorder equation with degree singularities and degenerations in coefficients by time and spatial of variables arbitrary order for some set of points. The existence and uniqueness of a solution of the problem is proved in Hölder spaces with degree weight.

Previous data. In paper [6] for a second-order parabolic equation problem with oblique derivative and impulse influence was reviewed. In paper [7], the problem of optimal control of a system described by the oblique derivativeproblem and the integral condition for a time variable for a second order parabolic equationwith power singularities in the equation and boundary condition coefficients is investigated. The coefficients of the equation and the boundary condition have power singulation arbitary order in the time space variables on some set of points. With the help of modified methods developed in the study of boundary-value problems forparabolic equations with smooth coefficients, a priori estimates, the existence and uniquenessof the solution of a nonlocal parabolic boundary-value problem with degeneracy was established.

This article is a continuation of research for nonlocal parabolic equations with degeneracy.

Problem statement. Let $\eta, t_{0}, t_{1}, \ldots, t_{N}, t_{N+1}$ be fixed positive numbers, $0 \leq t_{0}<t_{1}<\ldots<t_{N+1}$, $t_{0}<\eta<t_{N+1}, \eta \neq t_{\lambda}, \lambda \in\{1,2, \ldots, N\}$, let $\Omega$ be some bounded domain, $\bar{\Omega} \subset R^{n-1}, \operatorname{dim} \Omega \leq n-1$. Let
$\Pi_{(0)}=\left\{(t, x): t \in\left[t_{0}, t_{N+1}\right), x \in \Omega\right\} \bigcup\{(t, x): t=\eta$, $\left.x \in R^{n} \backslash \bar{\Omega}\right\}$. In the domain $\Pi=\left[t_{0}, t_{N+1}\right) \times R^{n}$ we consider a problem of finding the function $u(t, x)$, which, for $(t, x) \notin \Pi_{(0)}$ satisfies the equation

$$
\begin{gathered}
(L u)(t, x) \equiv\left[\partial_{t}-\sum_{i, j=1}^{n} A_{i j}(t, x) \partial_{x_{i}} \partial_{x_{j}}+\right. \\
\left.+\sum_{i=1}^{n} A_{i}(t, x) \partial_{x_{i}}+A_{0}(t, x)\right] u(t, x)=f(t, x),
\end{gathered}
$$

and condition for the time variable

$$
u\left(t_{0}+0, x\right)+\sum_{j=1}^{N} b_{j}(x) u\left(t_{j}, x\right)=\varphi(x) .
$$

The power singularities of coefficients of the differential equation (1) at the point $P(t, x) \in \Pi \backslash \Pi_{0}$ are characterized by functions $s_{1}\left(\beta_{i}^{(1)}, t\right)$ and $s_{2}\left(\beta_{i}^{(2)}, x\right)$ : $s_{1}\left(\beta_{i}^{(1)}, t\right)=|t-\eta|^{\beta_{i}^{(1)}} \quad$ if $\quad|t-\eta| \leq 1$, $s_{1}\left(\beta_{i}^{(1)}, t\right)=1 \quad$ if $\quad|t-\eta| \geq 1$, $s_{2}\left(\beta_{i}^{(2)}, x\right)=\rho^{\beta_{i}^{(2)}}(x)$ if $\rho(x) \leq 1, \quad s_{2}\left(\beta_{i}^{(2)}, x\right)=1$ if $\rho(x) \geq 1, \rho(x)=\inf _{z \in \Omega}|x-z|, \beta_{i}^{(v)} \in(-\infty, \infty)$, $v \in\{1,2\} \quad, \quad \beta^{(v)}=\left(\beta_{1}^{(v)}, \ldots, \beta_{n}^{(v)}\right)$ $\beta=\left(\beta^{(1)}, \beta^{(2)}\right)$.

Let's denote by $\left(x_{1}^{(1)}, \ldots, x_{i}^{(1)}, \ldots, x_{n}^{(1)}\right)$ the coordinates of the point $x^{(1)} \in R^{n}$, $\left(x_{1}^{(1)}, \ldots, x_{i-1}^{(1)}, x_{i}^{(2)}, x_{i+1}^{(1)}, \ldots, x_{n}^{(1)}\right)$ are the coordinates of the point $x^{(2)} \in R^{n}, \ell, \alpha, q^{(1)}, q^{(2)}, \gamma^{(1)}$, $\gamma^{(2)}, \mu_{j}^{(1)}, \mu_{j}^{(2)}$ are real numbers, $j \in\{0,1, \ldots, n\}$, $1 \geq 0, q^{(v)} \geq 0, \gamma^{(v)} \geq 0, \mu_{j}^{(v)} \geq 0, \alpha \in(0,1)$, [1] is the integer part of $l$. Let $D$ be an arbitrary closed domain, $\quad \bar{D} \subset R^{n}, \quad Q=\left[t_{0}, t_{N+1}\right) \times D \quad$, 


$$
P_{1}\left(t^{(1)}, x^{(1)}\right), \quad R\left(t^{(2)}, x^{(1)}\right), \quad H_{i}\left(t^{(1)}, x^{(2)}\right),
$$

$i \in\{1,2, \ldots, n\}$ are arbitrary points of the domain $Q$,

$\bar{Q} \subset \Pi=\left[t_{0}, t_{N+1}\right) \times R^{n}, Q_{(0)}=Q \cap \Pi_{(0)}$.

Let's define the functional space in which we study problem (1), (2).

$H^{1}(\gamma ; \beta ; q ; Q)$ is set of functions $u(t, x)$ of space $L_{1}(\Pi)$, which have continuous partial derivatives in $Q \backslash Q_{(0)}$ of the form $\partial_{t}^{j} \partial_{x}^{r}, \quad 2 i+|r| \leq[l]$ and a finite value of the norm

$$
\begin{aligned}
\|u ; \gamma ; \beta ; q ; Q\|_{l} & =\sum_{2 j+|r| \leq l}\|u ; \gamma ; \beta ; q ; Q\|_{2 j+|r|}+ \\
& +\langle u ; \gamma ; \beta ; q ; Q\rangle_{l},
\end{aligned}
$$

where, e.g.,

$$
\|u ; \gamma ; \beta ; 0 ; Q\|_{0}=\left(\sup _{P_{1} \in \bar{Q}}\left|u\left(P_{1}\right)\right|\right) \equiv\|u ; \Pi\|_{0},
$$

$$
\|u ; \gamma ; \beta ; q ; Q\|_{2 j+|r|} \equiv \sup _{P_{1} \in \bar{Q}}\left[s_{1}\left(q^{(1)}+2 j \gamma^{(1)}, t^{(1)}\right) s_{2}\left(q^{(2)}+2 j \gamma^{(2)}, x^{(1)}\right) \times\right.
$$$$
\times\left|\partial_{t}^{j} \partial_{x}^{r} u\left(P_{1}\right)\right| \prod_{i=1}^{n} s_{1}\left(r_{i}\left(\gamma^{(1)}-\beta_{i}^{(1)}\right), t^{(1)}\right) \times
$$$$
\left.\times s_{2}\left(r_{i}\left(\gamma^{(2)}-\beta_{i}^{(2)}\right), x^{(1)}\right)\right],
$$

$$
\langle u ; \gamma, \beta, q ; Q\rangle_{1}=\sum_{2 j+|r|=[1]}\left\{\sum _ { v = 1 } ^ { n } \operatorname { s u p } _ { ( P _ { 1 } , H _ { v } ) \subset \overline { Q } } \left[s_{1}\left(q^{(1)}+l \gamma^{(1)}, t^{(2)}\right) \times\right.\right.
$$$$
\times s_{2}\left(q^{(2)}+2 j \gamma^{(2)}, \tilde{x}\right) \prod_{i=1}^{n} s_{1}\left(-r_{i} \beta_{i}^{(1)}, t^{(1)}\right) s_{2}\left(r_{i}\left(\gamma^{(2)}-\beta_{i}^{(2)}, \tilde{x}\right)\right) \times
$$$$
\times s_{1}\left(-\{l\} \beta_{v}^{(1)}, t^{(1)}\right) s_{2}\left(\{1\}\left(\gamma^{(2)}-\beta_{v}^{(2)}, \tilde{x}\right)\right)\left|x_{v}^{(1)}-x_{v}^{(2)}\right|^{-\{1\}} \times
$$$$
\left.\times\left|\partial_{t}^{j} \partial_{x}^{r} u\left(P_{1}\right)-\partial_{t}^{j} \partial_{x}^{r} u\left(H_{v}\right)\right|\right]+
$$$$
+\sup _{\left(P_{1}, R\right) \subset \bar{Q}}\left[s_{1}\left(q^{(1)}+1 \gamma^{(1)}, \tilde{t}\right) s_{2}\left(q^{(2)}+(l-|r|) \gamma^{(2)}, x^{(2)}\right) \times\right.
$$

$$
\begin{gathered}
\times \prod_{i=1}^{n} s_{1}\left(-r_{i} \beta_{i}^{(1)}, \tilde{t}\right) s_{2}\left(r_{i}\left(\gamma^{(2)}-\beta_{i}^{(2)}, x^{(1)}\right)\right)\left|t^{(1)}-t^{(2)}\right|^{-\{1 / 2\}} \times \\
\left.\left.\times\left|\partial_{t}^{j} \partial_{x}^{r} u(R)-\partial_{t}^{j} \partial_{x}^{r} u\left(P_{1}\right)\right|\right]\right\} .
\end{gathered}
$$

Marked here:

$$
\begin{aligned}
& s_{1}(a, \tilde{t})=\min \left(s_{1}\left(a, t^{(1)}\right), s_{1}\left(a, t^{(2)}\right)\right), \\
& s_{2}(a, \tilde{x})=\min \left(s_{2}\left(a, x^{(1)}\right), s_{2}\left(a, x^{(2)}\right)\right), \\
& \partial_{x}^{r}=\partial_{x_{1}}^{r_{1}} \partial_{x_{2}}^{r_{2}} \ldots \partial_{x_{n}}^{r_{n}}, \quad|r|=r_{1}+\ldots+r_{n} .
\end{aligned}
$$

Let's assume that the initial date of problems (1), (2) satisfy the following conditions:

a) for the arbitrary vector $\xi=\left(\xi_{1}, \ldots, \xi_{n}\right) \forall(t, x) \in \Pi$ the following inequality holds

$$
\begin{gathered}
\pi_{1}|\xi|^{2} \leq \sum_{i, j=1}^{n} A_{i j}(t, x) s_{1}\left(\beta_{i}^{(1)}, t\right) s_{1}\left(\beta_{j}^{(1)}, t\right) \times \\
\times s_{2}\left(\beta_{i}^{(2)}, x\right) s_{2}\left(\beta_{j}^{(2)}, x\right) \xi_{i} \xi_{j} \leq \pi_{2}|\xi|^{2},
\end{gathered}
$$

$\pi_{1}, \pi_{2}$ are fixed positive constants and $s_{1}\left(\mu_{i}^{(1)}, t\right) s_{2}\left(\mu_{i}^{(2)}, x\right) A_{i} \in H^{\alpha}(\gamma ; \beta ; 0 ; \Pi)$ $s_{1}\left(\mu_{0}^{(1)}, t\right) s_{2}\left(\mu_{0}^{(2)}, x\right) A_{0} \in H^{\alpha}(\gamma ; \beta ; 0 ; \Pi), \quad A_{0} \geq 0$,

$s_{1}\left(\beta_{i}^{(1)}, t\right) s_{1}\left(\beta_{j}^{(1)}, t\right) s_{2}\left(\beta_{i}^{(2)}, x\right) s_{2}\left(\beta_{j}^{(2)}, x\right) A_{i j} \in$

$\in H^{\alpha}(\gamma ; \beta ; 0 ; Q)$

$\gamma^{(v)}=\max \left\{\max _{i}\left(1+\beta_{i}^{(v)}\right), \max _{i}\left(\mu_{i}^{(v)}-\beta_{i}^{(v)}\right), \frac{\mu_{0}^{(v)}}{2}\right\}$,

$v \in\{1,2\}$;

$$
\text { b) functions } \quad f \in H^{\alpha}\left(\gamma ; \beta ; \mu_{0} ; \Pi\right)
$$

$\varphi_{0} \in H^{2+\alpha}\left(\tilde{\gamma} ; \tilde{\beta}, 0 ; R^{n}\right) \quad, \quad b_{j}(x) \in C^{2+\alpha}\left(R^{n}\right) \quad$, $\sum_{j=1}^{N}\left|b_{j}(x)\right| \leq \lambda_{0}<1, \tilde{\gamma}=\left(0, \gamma^{(2)}\right), \tilde{\beta}=\left(0, \beta^{(2)}\right)$.

Main results. Let's formulate the main result of the work.

Theorem 1. Let conditions a), b) be satisfied for problem (1), (2). Then there exists a unique solution of problem (1), (2) in the space $H^{2+\alpha}(\gamma ; \beta ; 0 ; \Pi)$ and the following estimate is correct:

$$
\begin{gathered}
\|u ; \gamma ; \beta ; 0 ; \Pi\|_{2+\alpha} \leq c\left(\left\|f ; \gamma ; \beta ; \mu_{0} ; \Pi\right\|_{\alpha}+\right. \\
\left.+\left\|\varphi ; \tilde{\gamma} ; \tilde{\beta} ; 0 ; R^{n}\right\|_{2+\alpha}\right) \equiv B .
\end{gathered}
$$

To prove Theorem 1 we construct a sequence of solutions of problems with smooth coefficients, the boundary value of which is the solution of problem (1), (2).

Evaluation of solutions of problems with smooth coefficients. Let $\quad \Pi_{m}=\Pi \bigcap$ $\cap\left\{(t, x) \in \Pi: s_{1}(1, t) \geq m_{1}^{-1}, s_{2}(1, x) \geq m_{2}^{-1}\right\}, m=\left(m_{1}, m_{2}\right)$, $m_{1}>1, \quad m_{2}>1$ be a sequence of domains that, for $m_{1} \rightarrow \infty, m_{2} \rightarrow \infty$ converges to $\Pi \backslash \Pi_{(0)}$.

In the domain $\Pi$ we consider the problem of finding the function $u_{m}(t, x)$, that satisfies the equation

$$
\begin{aligned}
& \left(L_{1} u_{m}\right)(t, x) \equiv\left[\partial_{t}-\sum_{i, j=1}^{n} a_{i j}(t, x) \partial_{x_{i}} \partial_{x_{j}}+\right. \\
& \left.+\sum_{i=1}^{n} a_{i}(t, x) \partial_{x_{i}}+a_{0}(t, x)\right] u_{m}(t, x)=f_{m}(t, x),
\end{aligned}
$$

and condition for the time variable

$$
u_{m}\left(t_{0}+0, x\right)+\sum_{j=1}^{N} b_{j}(x) u_{m}\left(t_{j}, x\right)=\varphi_{m}(x) .
$$

Here, the coefficients $a_{i j}, a_{i}, a_{0}$ and functions $f_{m}$ , $\varphi_{m}$, for $(t, x) \in \Pi_{m}$ coincide with $A_{i j}, A_{i}, A_{0}$ and $f, \varphi$ respectively. For $(t, x) \in \Pi \backslash \Pi_{m}$ the coefficients $a_{i j}, a_{i}, a_{0}$, and functions $f_{m}, \varphi_{m}$ are continuous extensions of the coefficients $A_{i j}, A_{i}, A_{0}$ and functions $f_{m}, \varphi_{m}$ from the domain $\Pi_{m}$ into the domain $\Pi \backslash \Pi_{m}, m=\left(m_{1}, m_{2}\right)$.

Let us find the estimate of the solution to the problem 
(4), (5). In the space $C^{2+\alpha}(\Pi)$ we introduce the norm $\left\|u_{m} ; \gamma ; \beta ; q ; \Pi\right\|_{l}$, which, for every $m$ s equivalent to the Hölder norm determined by the same way as $\|u ; \gamma ; \beta ; q ; \Pi\|_{l}$, but instead of the functions $s_{1}\left(\beta_{i}^{(1)}, t\right)$ and $s_{2}\left(\beta_{i}^{(2)}, x\right)$ we take $d_{1}\left(\beta_{i}^{(1)}, t\right), d_{2}\left(\beta_{i}^{(2)}, x\right)$ : $d_{1}\left(\beta_{i}^{(1)}, t\right)=\max \left(s_{1}\left(\beta_{i}^{(1)}, t\right), m_{1}^{-\beta_{i}^{(1)}}\right)$ if $\beta_{i}^{(1)} \geq 0$ and $d_{1}\left(\beta_{i}^{(1)}, t\right)=\min \left(s_{1}\left(\beta_{i}^{(1)}, t\right), m_{1}^{-\beta_{i}^{(1)}}\right) \quad$ if $\quad \beta_{i}^{(1)}<0 ;$ $d_{2}\left(\beta_{i}^{(2)}, x\right)=\max \left(s_{2}\left(\beta_{i}^{(2)}, x\right), m_{2}^{-\beta_{i}^{(2)}}\right) \quad$ if $\quad \beta_{i}^{(2)} \geq 0 \quad$ and $d_{2}\left(\beta_{i}^{(2)}, x\right)=\min \left(s_{2}\left(\beta_{i}^{(2)}, x\right), m_{2}^{-\beta_{i}^{(2)}}\right)$ if $\beta_{i}^{(2)}<0$.

For the solution of the problem (4), (5) we have a correct theorem.

Theorem 2. Let $u_{m}$ be the classical solutions of problem (4), (5) in the domain $\Pi$ and let conditions a), b) be satisfied. Then for $u_{m}(t, x)$ the following estimate is true

$$
\left|u_{m}\right| \leq c\left(\left\|\varphi_{m}\left(1-\sum_{j=1}^{N}\left|b_{j}(x)\right|^{-1} ; R^{n}\right)\right\|_{0}+\left\|f_{m} ; \Pi\right\|_{0}\right) \text {. }
$$

Inequality (6) is proved by the scheme of proving of Theorem 2.5 [8, p. 27].

Theorem 3. If conditions a), b) are satisfied then for the solution of the problem (4), (5) the following inequality is true:

$$
\begin{gathered}
\left\|u_{m} ; \gamma, \beta, 0 ; \Pi\right\|_{2+\alpha} \leq c\left(\left\|f_{m} ; \gamma ; \beta ; \mu_{0} ; \Pi\right\|_{\alpha}+\right. \\
\left.+\left\|\varphi_{m} ; \tilde{\gamma} ; \tilde{\beta} ; 0 ; R^{n}\right\|_{2+\alpha}\right) .
\end{gathered}
$$

Proof. Using the definition of the norm and interpolation inequalities from $[9,10]$, we have

$$
\begin{gathered}
\left\|u_{m} ; \gamma ; \beta ; 0 ; \Pi\right\|_{2+\alpha} \leq\left(1+\varepsilon^{\alpha}\right)\left\langle u_{m} ; \gamma ; \beta ; 0 ; \Pi\right\rangle_{2+\alpha}+ \\
+c(\varepsilon)\left\|u_{m} ; \Pi\right\|_{0},
\end{gathered}
$$

where $\varepsilon$ is an arbitrary real number, $\varepsilon \in(0,1)$ This is why it suffices to evaluate the half norm $\left\langle u_{m} ; \gamma ; \beta ; 0 ; \Pi\right\rangle_{2+\alpha}$. From the definition of the half norm it follows that, in $\Pi$ there exist the points $P_{1}, R_{i}, H_{v}$, for which one of the following inequalities is true

$$
\frac{\lambda_{0}+1}{2}\left\|u_{m} ; \gamma ; \beta ; 0 ; \Pi^{(k)}\right\|_{2+\alpha} \leq E_{\delta}, \quad \delta \in\{1,2\}
$$

Where

$$
\begin{gathered}
E_{1} \equiv \sum_{2+|r|=2}\left\{\sum_{v=1}^{n} d_{1}\left((2+\alpha) \gamma^{(1)}, t^{(1)}\right) \times\right. \\
\times \prod_{i=1}^{n} d_{1}\left(-r_{i} \beta_{i}^{(1)}, t^{(1)}\right) d_{2}\left(r_{i}\left(\gamma^{(2)}-\beta_{i}^{(2)}\right), \tilde{x}\right) d_{1}\left(-\alpha \beta_{v}^{(1)}, t^{(1)}\right) \times \\
\times d_{2}\left(-\alpha\left(\gamma^{(2)}-\beta_{v}^{(2)}\right), \tilde{x}\right)\left|x_{v}^{(1)}-x_{v}^{(2)}\right|^{-\alpha} \times \\
\times\left|\partial_{t}^{j} \partial_{x}^{r} u_{m}\left(P_{1}\right)-\partial_{t}^{j} \partial_{x}^{r} u_{m}\left(H_{v}\right)\right|, \\
E_{2} \equiv \sum_{2 j+|r|=2} d_{1}\left((2+\alpha) \gamma^{(1)}, \tilde{t}\right) d_{2}\left((2-|r|+\alpha) \gamma^{(2)}, x^{(2)}\right) \times \\
\times \prod_{i=1}^{n} d_{1}\left(-r_{i} \beta_{i}^{(1)}, \tilde{t}\right) d_{2}\left(r_{i}\left(\gamma^{(2)}-\beta_{i}^{(2)}\right), x^{(2)}\right)\left|t^{(1)}-t^{(2)}\right|^{-\alpha / 2} \times
\end{gathered}
$$

$$
\begin{gathered}
\quad \times\left|\partial_{t}^{j} \partial_{x}^{r} u_{m}(R)-\partial_{t}^{j} \partial_{x}^{r} u_{m}\left(P_{1}\right)\right|, \\
d_{1}(a, \tilde{t})=\min \left(d_{1}\left(a, t^{(1)}\right), d_{2}\left(a, t^{(2)}\right)\right), \\
d_{2}(a, \tilde{x})=\min \left(d_{2}\left(a, x^{(1)}\right), d_{x}\left(a, x^{(2)}\right)\right) . \\
\quad \text { If }\left|x_{v}^{(1)}-x_{v}^{(2)}\right| \geq \frac{\varepsilon_{1}}{4} \frac{1}{n} d_{1}\left(\gamma^{(1)}-\beta_{v}^{(1)}, t^{(1)}\right) \times \\
\times d_{2}\left(\gamma^{(2)}-\beta_{v}^{(2)}, \tilde{x}\right) \equiv T_{1} \text { then } \\
E_{1} \leq 2 \varepsilon_{1}^{-\alpha}\left\|u_{m} ; \gamma ; \beta ; 0 ; \Pi\right\|_{2} . \\
\text { If }\left|t^{(1)}-t^{(2)}\right| \geq \frac{\varepsilon_{1}}{16} d_{1}\left(2 \gamma^{(1)}, \tilde{t}\right) d_{2}\left(2 \gamma^{(2)}, x^{(2)}\right) \equiv T_{2}
\end{gathered}
$$

then

$$
E_{2} \leq 2 \varepsilon_{1}^{-\alpha}\left\|u_{m} ; \gamma ; \beta ; 0 ; \Pi\right\|_{2} .
$$

Applying the interpolation inequalities to (9), (10), we obtain

$$
E_{\delta} \leq \varepsilon^{\alpha}\left\|u_{m} ; \gamma ; \beta ; 0 ; \Pi\right\|_{2+\alpha}+c(\varepsilon)\left\|u_{m} ; \Pi\right\|_{0} .
$$

Let $\left|x_{v}^{(1)}-x_{v}^{(2)}\right| \leq T_{1}$, and $\left|t^{(1)}-t^{(2)}\right| \leq T_{2}$. We assume that $\quad d_{1}\left(2 \gamma^{(1)}, \tilde{\tau}\right)=d_{1}\left(2 \gamma^{(1)}, t^{(1)}\right)$, $d_{2}\left(2 \gamma^{(2)}, \tilde{x}\right)=d_{2}\left(2 \gamma^{(2)}, x^{(1)}\right),\left(t^{(1)}, x^{(1)}\right) \in \Pi$. In the domain $\Pi$ we write problem (4), (5) in the form

$$
\begin{gathered}
\left(L_{2} u_{m}\right) \equiv\left[\partial_{t}-\sum_{i, j=1}^{n} a_{i j}\left(t^{(1)}, x^{(1)}\right) \partial_{x_{i}} \partial_{x_{j}}\right] u_{m}= \\
=\sum_{i, j=1}^{n}\left[a_{i j}(t, x)-a_{i j}\left(t^{(1)}, x^{(1)}\right)\right] \partial_{x_{i}} \partial_{x_{j}} u_{m}- \\
-\sum_{i=1}^{n} a_{i}(t, x) \partial_{x_{i}} u_{m}-a_{0}(t, x) u_{m}+f_{m}(t, x) \equiv \\
\equiv F_{m}\left(t, x ; u_{m}\right)+f_{m}(t, x), \\
u_{m}\left(t_{0}+0, x\right)=\psi_{m}\left(t_{0}, x\right),
\end{gathered}
$$

where $\psi_{m}\left(t_{0}, x\right)=\varphi_{m}(x)-\sum_{j=1}^{N} u_{m}\left(t_{j}, x\right)$.

Let $V_{\varepsilon_{2}}$ be a domain from $\Pi$, $V_{\varepsilon_{2}}=\left\{(t, x) \in \Pi,\left|t-t^{(1)}\right| \leq \varepsilon_{2}^{2} T_{2},\left|x_{i}-x_{i}^{(1)}\right| \leq \varepsilon_{2} T_{1}\right.$, $i \in\{1, \ldots, n\}\}$. In problem (12), (13) we make the substitution $\quad u_{m}(t, x)=v_{m}(t, x)$

$y_{i}=d_{1}\left(\beta_{i}^{(1)}, t^{(1)}\right) d_{1}\left(\beta_{i}^{(2)}, x^{(1)}\right) x_{i}$. As a result, we are obsessed

$$
\begin{gathered}
\left(L_{2} v_{m}\right)(t, y) \equiv\left[\partial_{t}-\sum_{i, j=1}^{n} d_{1}\left(\beta_{i}^{(1)}, t^{(1)}\right) d_{1}\left(\beta_{j}^{(1)}, t^{(1)}\right) \times\right. \\
\left.\times d_{2}\left(\beta_{i}^{(2)}, x^{(1)}\right) d_{2}\left(\beta_{j}^{(2)}, x^{(1)}\right) a_{i j}\left(t^{(1)}, x^{(1)}\right) \partial_{y_{i}} \partial_{y_{j}}\right] v_{m}= \\
=F_{m}\left(t, \tilde{y} ; v_{m}\right)+f_{m}(t, \tilde{y}), \\
v_{m}\left(t_{0}+0, y\right)=\psi_{m}\left(t_{0}, y\right), \\
\text { where } \tilde{y}=\left(d_{1}\left(-\beta_{1}^{(1)}, t^{(1)}\right) d_{2}\left(-\beta_{1}^{(2)}, x^{(1)}\right) y_{1}, \ldots,\right. \\
\left.d_{1}\left(-\beta_{n}^{(1)}, t^{(1)}\right) d_{2}\left(-\beta_{n}^{(2)}, x^{(1)}\right) y_{n}\right) . \\
\text { Let's denote } y_{i}^{(1)}=d_{1}\left(\beta_{i}^{(1)}, t^{(1)}\right) d_{2}\left(\beta_{i}^{(2)}, x^{(1)}\right) x_{i}^{(1)},
\end{gathered}
$$




$$
W_{\varepsilon_{2}}=\left\{(t, y),\left|t-t^{(1)}\right| \leq \varepsilon_{2}^{2} T_{2},\left|y_{i}-y_{i}^{(1)}\right| \leq \varepsilon_{2} \sqrt{T_{2}}\right\}
$$

and take the triply differentiable function $\mu(t, y)$ that satisfies the conditions

$$
\mu(\tau, z)=\left\{\begin{array}{lll}
1, & (t, y) \in W_{1 / 2}, & 0 \leq \mu(t, y) \leq 1, \\
0, & (t, y) \notin W_{3 / 4}, & \left|\partial_{t}^{j} \partial_{x}^{r} \mu\right| \leq c_{j r} \times \\
& \times d_{1}\left(-2 j \gamma^{(1)}, t^{(1)}\right) \times \\
& \times \prod_{i=1}^{n} d_{2}\left(-r_{j} \gamma^{(2)}, x^{(2)}\right) .
\end{array}\right.
$$

Then the function $Z_{m}(t, y)=\mu(t, y) v_{m}(t, y)$ is a solution of the Cauchy problem

$$
\begin{gathered}
\left(L_{2} Z_{m}\right)(t, y)=\sum_{i, j=1}^{n} d_{1}\left(\beta_{i}^{(1)}, t^{(1)}\right) d_{1}\left(\beta_{j}^{(1)}, t^{(1)}\right) \times \\
\times d_{2}\left(\beta_{i}^{(2)}, x^{(1)}\right) d_{2}\left(\beta_{j}^{(2)}, x^{(1)}\right) a_{i j}\left(t^{(1)}, x^{(1)}\right) \times \\
\times\left[\partial_{y_{i}} \mu \partial_{y_{j}} v_{m}+\partial_{y_{j}} \mu \partial_{y_{i}} v_{m}\right]+ \\
+v_{m}\left[\sum_{i, j=1}^{n} d_{1}\left(\beta_{i}^{(1)}, t^{(1)}\right) d_{1}\left(\beta_{j}^{(1)}, t^{(1)}\right) d_{2}\left(\beta_{i}^{(2)}, x^{(1)}\right) \times\right. \\
\left.\times d_{2}\left(\beta_{j}^{(2)}, x^{(1)}\right) a_{i j}\left(t^{(1)}, x^{(1)}\right) \partial_{y_{i}} \partial_{y_{j}} \mu-\partial_{t} \mu\right]+ \\
+\mu\left[F_{m}+f_{m}\right] \equiv F_{m}^{(1)}+\mu f_{m}, \\
Z_{m}\left(t_{0}+0, x\right)=\psi_{m}\left(t_{0}, \tilde{y}\right) \mu\left(t_{0}, y\right) .
\end{gathered}
$$

On the basis of Theorem 5.1 from [8, p. 364], for the solution of problem (16), (17) the following inequalities are true:

$$
\begin{gathered}
\left|y^{(1)}-y^{(2)}\right|^{-\alpha}\left|\partial_{t}^{j} \partial_{y}^{r} Z_{m}\left(t, y^{(1)}\right)-\partial_{t}^{j} \partial_{y}^{r} Z_{m}\left(t, y^{(2)}\right)\right| \leq \\
\leq c\left(\left\|F_{m}^{(1)}+\mu F_{m}\right\|_{C^{\alpha}\left(W_{3 / 4}\right)}+\right. \\
\left.+\left\|\mu \psi_{m}\right\|_{C^{2+\alpha}\left(W_{3 / 4} \cap\left(t=t_{0}\right)\right)}\right) \equiv B_{m}, \\
\left|t^{(1)}-t^{(2)}\right|^{-\alpha / 2}\left|\partial_{t}^{j} \partial_{y}^{r} Z_{m}\left(t^{(1)}, y\right)-\partial_{t}^{j} \partial_{y}^{r} Z_{m}\left(t^{(2)}, y\right)\right| \leq \\
\leq c_{1} B_{m},
\end{gathered}
$$

where $\left\{\left(t, y^{(1)}\right),\left(t, y^{(2)}\right),\left(t^{(1)}, y\right),\left(t^{(2)}, y\right)\right\} \subset W_{1 / 4}$, $2 j+|r|=2$.

Taking into account the properties of the function $\mu(t, y)$ we obtain

$$
\begin{gathered}
\left\|F_{m}^{(1)}+\mu f_{m}\right\|_{C^{\alpha}\left(W_{3 / 4}\right)} \leq c_{2} d_{1}\left(-(2+\alpha) \gamma^{(1)}, t^{(1)}\right) \times \\
\times d_{2}\left(-(2+\alpha) \gamma^{(2)}, x^{(1)}\right)\left(\left\|v_{m} ; \gamma ; 0,0 ; W_{3 / 4}\right\|_{2}+\right. \\
\left.\quad+\left\|v_{m} ; W_{3 / 4}\right\|_{0}+\left\|F_{m} ; \gamma ; 0,2 \gamma ; W_{3 / 4}\right\|_{\alpha}\right), \\
\left\|\mu \psi_{m}\right\|_{C^{2+\alpha}\left(W_{3 / 4} \cap\left(t=t_{0}\right)\right)} \leq c_{3} d_{1}\left(-(2+\alpha) \gamma^{(1)}, t^{(1)}\right) \times \\
\times d_{2}\left(-(2+\alpha) \gamma^{(2)}, x^{(1)}\right)\left\|\psi_{m} ; \tilde{\gamma} ; 0 ; 0 ; W_{3 / 4}\right\|_{2+\alpha}, \quad(20)
\end{gathered}
$$

Substituting (19), (20) into (18) and returning to the variables $(t, x)$, we obtain

$$
\begin{gathered}
E_{\delta} \leq c_{4}\left(\left\|F_{m} ; \gamma, \beta ; 2 \gamma ; V_{3 / 4}\right\|_{\alpha}+\left\|f_{m} ; \gamma, \beta ; 2 \gamma ; V_{3 / 4}\right\|_{\alpha}+\right. \\
+\left\|u_{m} ; V_{3 / 4}\right\|_{0}+\left\|u_{m} ; \gamma, \beta ; 0 ; V_{3 / 4}\right\|_{2}+ \\
\left.+\left\|\psi_{m} ; \tilde{\gamma}, \tilde{\beta} ; 0 ; V_{3 / 4}\right\|_{2+\alpha}\right) .
\end{gathered}
$$

Given the interpolation inequalities and estimates of the norm of each additive of the

expressions $F_{m}, \psi_{m}$, we obtain the inequalities

$$
\begin{gathered}
E_{\delta} \leq\left(\varepsilon_{1}^{\alpha}(n+2)+\varepsilon^{2} n^{2}\right)\left\|u_{m} ; \gamma ; \beta ; 0 ; V_{3 / 4}\right\|_{2+\alpha}+ \\
+c_{4}\left\|u_{m} ; V_{3 / 4}\right\|_{0}+c_{5}\left(\left\|f_{m} ; \gamma, \beta ; \mu_{0} ; V_{3 / 4}\right\|_{\alpha}+\right. \\
\left.+\left\|\psi_{m} ; \tilde{\gamma}, \tilde{\beta} ; 0 ; V_{3 / 4}\right\|_{2+\alpha}\right),
\end{gathered}
$$

where

$$
\begin{gathered}
\left\|\psi_{m} ; \tilde{\gamma}, \tilde{\beta} ; 0 ; V_{3 / 4}\right\|_{2+\alpha} \leq c\left(\left\|\varphi_{m} ; \tilde{\gamma}, \tilde{\beta} ; 0 ; R^{n}\right\|_{2+\alpha}+\right. \\
\left.+\lambda_{0}\left\|u_{m} ; \gamma, \beta ; 0 ; V_{3 / 4}\right\|_{2+\alpha}\right) .
\end{gathered}
$$

Using inequalities (6), (8), (11), (21), (22) and choosing $\varepsilon$ and $\varepsilon_{1}$ sufficiently small, we obtain

the estimate (7).

Theorem is proved.

Now let's establish the existence of a solution to the problem (4), (5).

Such a theorem is correct.

Theorem 3. If the conditions a), b) are satisfied then the unique solution to the problem (4), (5) exists for which the estimate (3) is valid.

The solution of the problem (4), (5) is constructed in the form

$$
u_{m}(t, x)=\int_{R^{n}} Z_{m}\left(t, x, t_{0}, \xi\right) u_{0}\left(t_{0}, \xi\right) d \xi+\omega_{m}(t, x),
$$

Where $\omega_{m}(t, x)$ is the solution of the Cauchy problem

$$
\left(L_{1} \omega_{m}\right)(t, x)=f_{m}(t, x), \omega_{m}\left(t_{0}+0, x\right)=\varphi_{m}(x), \quad(23)
$$
where $Z_{m}(t, x, \tau, \xi)$ is fundamental solution of the problem (23). Satisfying the condition (5), we obtain

$$
\begin{gathered}
u_{m}\left(t_{0}+0, x\right)+\sum_{j=1}^{N} b_{j}(x) \int_{R^{n}} Z_{m}\left(t_{j}, x, t_{0}, \xi\right) u_{m}\left(t_{0}, \xi\right) d \xi= \\
=-\sum_{j=1}^{N} b_{j}(x) \omega_{m}\left(t_{j}, x\right) .
\end{gathered}
$$

Given the restrictions on functions $b_{j}(x)$ by the method of successive approximations the solution of the integral equation (24) is constructed. Substituting it into (23) we obtain the solution of the problem (4), (5) for which the estimate (7) is valid.

Proof of Theorem 1. Because

$$
\begin{aligned}
& \left\|f_{m} ; \gamma ; \beta ; \mu_{0} ; \Pi\right\|_{\alpha} \leq c\left\|f ; \gamma ; \beta ; \mu_{0} ; \Pi\right\|_{\alpha}, \\
& \left\|\varphi_{m} ; \tilde{\gamma} ; \tilde{\beta} ; 0 ; R^{n}\right\|_{2+\alpha} \leq c\left\|\varphi ; \tilde{\gamma} ; \tilde{\beta} ; 0 ; R^{n}\right\|_{2+\alpha},
\end{aligned}
$$

then based on the estimate (7) for the solution of the problem (4), (5) the following estimate is true

$$
\left\|u_{m} ; \gamma ; \beta ; 0 ; \Pi\right\|_{2+\alpha} \leq B
$$

the right-hand side of inequality does not depend on $m=\left(m_{1}, m_{2}\right)$. Moreover, the sequences 


$$
\begin{aligned}
& \left\{U_{m}^{(0)}\right\} \equiv\left\{u_{m}\right\}, \\
& \left\{U_{m}^{(1)}\right\} \equiv\left\{d_{1}\left(\gamma^{(1)}, t\right) d_{2}\left(\gamma^{(2)}-\beta_{i}^{(2)}, x\right) \partial_{x_{i}} u_{m}(\mathrm{t}, \mathrm{x})\right\}, \\
& \left\{U_{m}^{(2)}\right\} \equiv\left\{d_{1}\left(2 \gamma^{(1)}, t\right) d_{2}\left(\gamma^{(2)}-\beta_{i}^{(2)}, x\right) \times\right. \\
& \left.\times d_{2}\left(\gamma^{(2)}-\beta_{j}^{(2)}, x\right) \partial_{x_{i}} \partial_{x_{j}} u_{m}(\mathrm{t}, \mathrm{x})\right\}, \\
& \left\{U_{m}^{(3)}\right\} \equiv\left\{d_{1}\left(2 \gamma^{(1)}, t\right) d_{2}\left(2 \gamma^{(2)}, x\right) \partial_{t} u_{m}(\mathrm{t}, \mathrm{x})\right\}
\end{aligned}
$$

are uniformly bounded and equicontinuous in the domain $\bar{Q}$. According to the Arzela theorem, there exist subsequences $\left\{U_{m_{k}}^{(v)}\right\}$, uniformly convergent in $\bar{Q}$ to $\left\{U_{0}^{(v)}\right\}, v \in\{0,1,2,3\}$. Since $Q$ is an arbitrary domain,
$Q \subset \Pi$, then passing to the limit for $m_{1 k} \rightarrow \infty$, $m_{2 k} \rightarrow \infty$ in problem (4), (5), we find that $u(t, x)=U_{0}^{(0)}-$ is the unique solution to the problem (1), (2), in the space $H^{2+\alpha}(\gamma ; \beta ; 0 ; \Pi)$. Theorem is proved.

Conclusions. The necessary and sufficient conditions for the existence of the unique solution of a multipoint problem for parabolic equations with degeneration are established. Estimates of derivatives

of the solution of the problem in the Hölder spaces with power weight are found. The order of the degree weight depends on the power of the degree features of the coefficients of the equation.

\section{REFERENCES}

1. A.M. Nakhushev. Equations of mathematical biology [in Russian], Higher School, Moscow (1995).

2. Belovich I.A., Kapitsa S.P., Kordyumov S.P. Mathematical model of global demographic processes taking into account spatial distribution [in Russian], Journal computational mathematics and mathematical physics, (1988), Vol. 38, N6.

3. Maykov A.R., Poyezd A.D., Yakunin S.A. The economic method of calculating nonstationary time-non-local radiation conditions for wave systems [in Russian] Journal computational mathematics and mathematical physics, (1980), Vol. 30, N8.

4. Seitz F. The modern theory of solids. - L.M. Gostekhizdat (1949)

5. Jasmine G.M. On the finiteness of the discrete spectrum of the energy operators of quantum systems of many particles [in Russian] Doc. SSSR Academy of Sciences, (1972), Vol. 207, N1.
6. I. D. Pukalskyi, B. O. Yashan Boundary-value problem with impulsive action for a parabolic equation with degeneration [in Ukrainian] Ukrainian Mathematical Journal (2019), Vol. 71, N1.

7. I.D. Pukal'skii, B.O. Yashan Optimal control in a nonlocal boundary value problem with integral condition for parabolic equations with degeneration [in Ukrainian], Bukovinian Math. Journal (2019), Vol. 7 N1.

8. Ladyzhenskaya O. A., Solonnikov V. A., Ural'tseva N. N. Linear and quasilinear equations of parabolic type [in Russian], Science, Moscow (1967).

9. Friedman A. Partial differential equations of parabolic type [in Russian], World, Moscow (1968).

10. Pukalskyi I.D. The boundary value problems for unevenly parabolic and elliptic equations with degeneration and singularities [in Ukrainian], Ruta, Chernivtsi (2008). 